\title{
Wave energy dissipation by phase mixing in magnetic coronal plasmas
}

\author{
I. J. D. Craig and G. Fruit
}

\author{
University of Waikato, New Zealand \\ e-mail: fruit@waikato.ac.nz \\ Received 4 March 2005 / Accepted 2 May 2005
}

\begin{abstract}
Wave energy dissipation by viscous and resistive damping in magnetic coronal plasmas is examined. We begin by pointing out that since the dimensionless viscous damping coefficient $v$ is generally much greater than the dimensionless resistivity $\eta$, viscous dissipation can be expected to be the dominant mechanism in many coronal applications. A detailed analysis is presented for the case of perpendicular polarized shear wave disturbances which propagate in a horizontally stratified magnetic channel. We show that when the equilibrium field contains a neutral line (specifically, $\boldsymbol{B}=B_{0} y \widehat{\boldsymbol{x}}$ ) the development of small length scales by phase mixing $\ell \simeq(\eta+v)^{1 / 3}$ leads to efficient wave energy losses: in particular, the bulk of the energy losses take place over the time interval $(\eta+v)^{-1 / 3}$. However, the later stages of the decay depends critically on whether viscosity or resistivity provides the dominant damping mechanism. When resistivity is sufficiently small the decay rate weakens at later times due to the emergence of a self-similar mode which allows a separation of the global kinetic and magnetic energies $(v \gg \eta)$. If the resistivity is large enough but still not dominant, self-similar behaviour can give way to monotonic exponential damping on the visco-resistive length scale $\ell \simeq(\eta v)^{1 / 6}$. In either case, provided only that $v>\eta$, energy equipartition eventually breaks down and the remnants of the initial wave energy wind up mainly in the magnetic field.
\end{abstract}

Key words. Sun: corona - magnetohydrodynamics (MHD) - waves

\section{Introduction}

The role of viscous effects in the magnetic energy dissipation of coronal plasmas has not been well studied. Most investigations focus on resistive release mechanisms which involve changes in the field topology due to magnetic reconnection. Indeed, since magnetic energy bound up in the global field topology can be released only by reconnection, resistive effects seem to provide a royal road for understanding the large scale structure of coronal magnetic fields (see e.g. Priest \& Forbes 2000).

One difficulty that plagues reconnection studies, however, is the low resistivities of typical coronal plasmas. The fact that the dimensionless resistivity $\eta$ is an inverse Lundquist number of order $10^{-14}$ (Spitzer 1962), makes it very difficult to construct mechanisms that achieve physically significant energy release. Even when fast reconnection is formally demonstrated - for instance in the cold, line-tied, $X$-points of Craig \& McClymont (1991, 1993) and Hassam (1992) - the effects of finite plasma pressure, can easily undo the strong field localizations required to make the mechanism effective $\left(\ell \leq \eta^{1 / 2}\right)$. In this respect viscous dissipation, whose effects are not burdened by such weak damping coefficients, should be more robust $(v \gg \eta)$. Viscous damping cannot alter the global field topology but, acting alone or in tandem with the plasma resistivity, it could provide an efficient and robust energy release mechanism, of interest in both rapid flare release and quiescent coronal heating.

In fact Hollweg (1986) has already emphasized theoretically that viscosity could be important in a wide variety of coronal processes. The combined effects of viscosity and resistivity also played a key role in the pioneering phase-mixing study of Heyvaerts \& Priest (1983) and in subsequent investigations (Hood et al. 1997a,b; Ruderman et al. 1998). A central result emerging from these studies is that phase mixing induces strong spatial gradients which lead to enhanced viscous and resistive dissipation on time scales of the order of $(v+\eta)^{-1 / 3}$.

Our present aim is to extend these studies by concentrating on visco-resistive damping in the vicinity of a magnetic null point. Although our ultimate goal is to study energy dissipation in general $X$-point field geometries, we concentrate for the present on Alfvén wave damping in a 1D neutral sheet. This simple configuration allows us to demonstrate analytically how visco-resistive phase mixing can affect the global energy dissipation rate. We find that although the initial damping phase is in good agreement with the previous phase mixing studies, the presence of a null point in the background magnetic field leads to several new features in the later decay. In particular, fast initial dissipation may be followed by either oscillatory decay (for $\eta>v$ ), or a more slowly decaying asymptotic tail due to selfsimilar behaviour or the emergence of a visco-resistive scale 
in the vicinity of the null point $(\eta<v)$. Our arguments suggest that similar conclusions may go through in more general $X$-point configurations.

In Sect. 2 we introduce the $2+1 / 2$ dimensional MHD geometry that provides the backdrop for our visco-resistive calculations. Numerical results are presented in Sect. 2.6 which illustrate typical features of the shear wave energy decay. Section 3 is concerned with the theoretical interpretation of these results and the development of scaling laws that summarize the distinct phases of the energy losses. We go on to show that in the physically realistic case where $v \gg \eta$, the visco-resistive coupling leads to a breakdown of energy equipartition in the later stages of the decay.

\section{Shear waves dissipation in magnetic $X$-points}

\subsection{Introduction}

We consider an incompressible magnetic plasma in planar geometry in which energy losses occur by viscous and resistive dissipation. By using a stream function representation for the plasma velocity,

$v(x, y, t)=\nabla \phi \times \hat{z}+W \hat{z}$,

and a flux function representation for the magnetic field,

$\boldsymbol{B}(x, y, t)=\nabla \psi \times \hat{z}+Z \hat{z}$,

we identically satisfy the divergence-free conditions for $\boldsymbol{v}$ and $\boldsymbol{B}$.

It is convenient to adopt a dimensionless formulation in which the magnetic field intensities, particle densities and lengths are scaled according to reference coronal values. Velocities and time are then measured in units of the Alfvén speed $v_{\mathrm{A}}$ and the Alfvén time $\tau_{\mathrm{A}}$ respectively. For a coronal plasma of length scale $10^{9.5} \mathrm{~cm}$, number density $10^{9} \mathrm{~cm}^{-3}$ and field $10^{2} \mathrm{G}$, we find that $v_{\mathrm{A}}=10^{9} \mathrm{~cm} \mathrm{~s}^{-1}$ and $\tau_{\mathrm{A}} \simeq 3 \mathrm{~s}$.

\subsection{Planar MHD equations}

The equations governing the evolution of the $\boldsymbol{v}$ and $\boldsymbol{B}$-fields are the non-dimensionalized momentum and induction equations. These are conveniently written using the Poisson bracket notation $[\psi, \phi]=\psi_{x} \phi_{y}-\psi_{y} \phi_{x}$, where $\psi_{x}$ denotes $\partial \psi / \partial x$ etc. The evolution equations reduce to

$$
\begin{aligned}
& \left(\nabla^{2} \phi\right)_{t}+\left[\nabla^{2} \phi, \phi\right]=\left[\nabla^{2} \psi, \psi\right]+v \nabla^{4} \phi, \\
& \psi_{t}+[\psi, \phi]=\eta \nabla^{2} \psi, \\
& W_{t}+[W, \phi]=[Z, \psi]+v \nabla^{2} W, \\
& Z_{t}+[Z, \phi]=[W, \psi]+\eta \nabla^{2} Z,
\end{aligned}
$$

on adopting simple Laplacian forms for the resistive and viscous dissipation terms.

Of particular importance is the smallness of $\eta$, an inverse Lundquist number of order $10^{-14}$. Although the dimensionless viscosity $v \simeq 10^{-4.5}$ is considerably larger, we should bear in mind that in a strongly magnetized plasma, the viscosity is a highly non-isotropic tensor (Hollweg 1986; Braginskii 1965). The form we adopt is justified only if the magnetic field in the

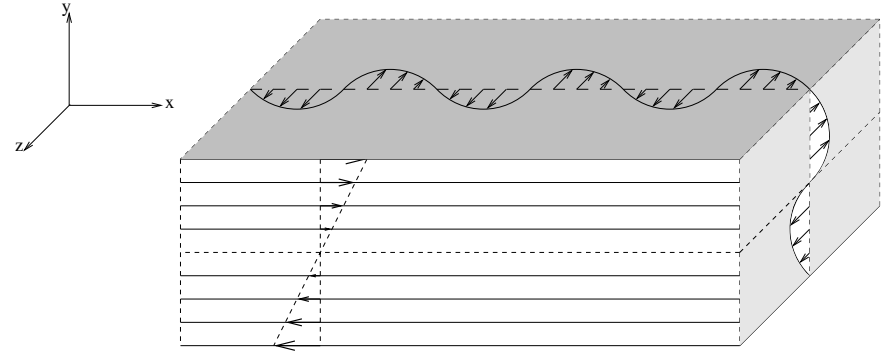

Fig. 1. The geometry of the background magnetic field: $\boldsymbol{B}=\hat{y} \widehat{\boldsymbol{x}}$ and sketch of the shear disturbance propagating along the magnetic field lines.

dissipation region is sufficiently weak. As illustrated in Fig. 1, the geometry we consider contains a weak field region surrounding the neutral line $y=0$, in which the isotropic viscosity should be a sensible approximation. This geometry provides possibly the simplest model of wave dissipation consistent with phase mixing in the presence of viscous and resistive effects.

\subsection{Perpendicular polarized shear-wave disturbances}

As outlined in Fig. 1, we assume that axial disturbances are superposed onto a one dimensional background field $\boldsymbol{B}=y \widehat{\boldsymbol{x}}$ with vanishing background flow ( $\psi=y^{2} / 2$ and $\phi=0$ respectively). This field supports perpendicularly polarized shear wave disturbances $Z(x, y, t)$ and $W(x, y, t)$ which using Eqs. (5) and (6) evolve according to

$W_{t}=y Z_{x}+v \nabla^{2} W$

$Z_{t}=y W_{x}+\eta \nabla^{2} Z$

These equations form the basis of our analysis. From these it is straightforward to derive an expression for the global energy losses of the system. If $\mathcal{E}$ is the global energy of the field $\left(W^{2}+\right.$ $\left.Z^{2}\right) / 2$ then

$\mathcal{E}_{t}=\int v\left[\left(W_{x}\right)^{2}+\left(W_{y}\right)^{2}\right]+\eta\left[\left(Z_{x}\right)^{2}+\left(Z_{y}\right)^{2}\right] \mathrm{d} V$.

Although collisional plasmas generally satisfy $v>\eta>0$, our present purpose is to study the system and quantify the energy losses, using a mixture of analytical and numerical techniques for small but arbitrary $v$ and $\eta$.

\subsection{Fourier decomposition in $x$}

Since the system (7)-(8) is homogeneous in the $x$-direction, it is expedient, for both analytic and numerical purposes, to filter out the explicit $x$-dependence by letting

$\left(\begin{array}{c}W(x, y, t) \\ Z(x, y, t)\end{array}\right)=\mathrm{e}^{\mathrm{i} m x}\left(\begin{array}{c}W(y, t) \\ i Z(y, t)\end{array}\right)$.

To avoid cluttering the notation we shall rely only on context to distinguish between the field $W(x, y, t)$ and its Fourier representation $W(y, t)$. Hence, the above system may be rewritten as

$W_{t}+m y Z=v\left(W_{y y}-m^{2} W\right)$,

$Z_{t}-m y W=\eta\left(Z_{y y}-m^{2} Z\right)$ 
The wave number $m$ will take discrete values if there are boundary conditions along the $x$-direction or continuous values if the channel is open and the shear Alfvén waves are free to propagate towards $\pm \infty$. Both situations are covered by the following analysis. Before considering specific solutions of (11)-(12) we briefly mention the role that phase mixing is likely to play in the damping of the system.

\subsection{Ideal solutions}

Consider first the case where both resistivity and viscosity are absent in the system. Each field line - parameterized by its vertical position $y=y_{\mathrm{p}}$ - is now uncoupled from its neighbour. The ideal system (11)-(12) has the solution

$$
\begin{aligned}
W & =A \cos \left(m y_{\mathrm{p}} t+\chi\right), \\
Z & =A \sin \left(m y_{\mathrm{p}} t+\chi\right),
\end{aligned}
$$

and we note that the oscillation frequency $\omega=m y_{\mathrm{p}}$, as well as the amplitude and phase, vary from line to line. The total energy $\mathcal{E}$ is clearly preserved $\left(W^{2}+Z^{2}=1\right)$, but there is a continual exchange of magnetic and kinetic energies in the system.

The fact that the frequency $\omega$ depends continuously on $y_{\mathrm{p}}$ can be interpreted in two ways:

- if the system is closed in the $x$-direction and the field lines are held fixed on some boundary at $x= \pm L$, the above solution describes a set of standing waves with discrete wave numbers $m=n \pi / L$. The differing oscillation rates lead to a phase shifts between neighbouring field lines.

- if the system is unbounded in the $x$-direction, the solution (13)-(14) represent a travelling Alfvén wave at a velocity $v_{\mathrm{A}}=y_{\mathrm{p}}$ (in units of the reference Alfvén speed $v_{\mathrm{A}}$ ). In this case a phase shift between two neighbouring field lines arises due to the differing wave propagation speeds.

In both situations, phase-shifts between the field lines lead to cross-gradients $W_{y}$ and $Z_{y}$ that grow linearly in time whereas the gradients along the field remain bounded. Consider, for example, the viscous damping term $v\left(W_{x}\right)^{2}$ in (9) that derives from gradients along the field lines. This is limited by the decay of the fundamental mode and clearly $\left|v m^{2}\right| \ll 1$ for $m \simeq 1, v \ll 1$. It follows that the $x$-dependance in the dissipation terms can generally be neglected. Conversely, the damping due to $v\left(W_{y}\right)^{2}$ grows as $m^{2} t^{2}$ and becomes important on the time scale $v^{-1 / 2}$, even for $m \simeq 1$. It is this effect that we wish to quantify in the following phase-mixing analysis.

\subsection{Numerical examples of phase mixing}

For reasons discussed in the appendix, it does not seem possible to obtain a global analytic solution to the system (7)-(8) valid for arbitrary $v$ and $\eta$. Our approach is therefore to simulate the system numerically and seek analytic solutions only in certain special cases. For the purposes of computation we assume that both $Z$ and $W$ vanish on the upper and lower surfaces of the channel $y= \pm 1$. We use the modal field representation (10) and solve the system (11)-(12) using an explicit predictor-corrector numerical scheme based on second order differences. In the

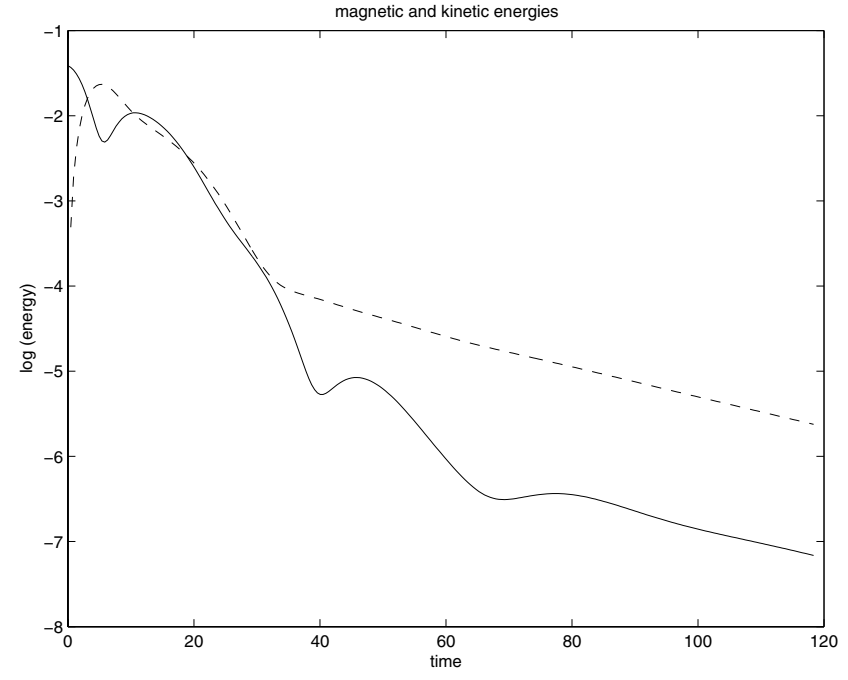

Fig. 2. The solid line shows the decay of the global kinetic energy in the case $\eta=10^{-4}, v=10^{-3}$. The kinetic component separates from the magnetic energy (dashed line) at around thirty Alfvén times. A phase of slow exponential damping sets in at around eighty Alfvén times.

results presented below we take $m=1$ and choose the initial conditions of the form

$W(y, 0)=\sin (\pi y), \quad Z(y, 0)=0$.

Note that, although we always set

$W( \pm 1, t)=Z( \pm 1, t)=0$,

the initial conditions ensure that the velocity field is an odd function of $y$ while the magnetic field is even. In particular, $Z$ at the origin is generally non-vanishing for $\eta>0$.

Figure 2 shows the global magnetic and kinetic energies against time in the case $v=10^{-3}, \eta=10^{-4}$. Three phases may be distinguished:

1. An initial stage of fast energy decay persists until about 30 Alfvèn times. Energy equipartition is well represented in this phase.

2. A transition phase occurs around 30 Alfvèn times. The most visible manifestation is the marked separation in the energy plots, which derives from the preferential damping of the kinetic energy.

3. An asymptotic phase of slow exponential damping takes place at roughly 80 Alfvèn times.

In Fig. 3 we consider the case of dominant of resistivity $\eta=$ $10^{-3} v=10^{-4}$. While the initial stage is similar to that of Fig. 2, the later decay - after about 40 Alfvén times - is utterly different. This phase is consistent with an oscillatory exponential decline in which equipartition is maintained by a continual interchange of magnetic and fluid energies. Conspicuously absent are the distinct phases of Fig. 2 associated with the separation of magnetic and kinetic energies. 


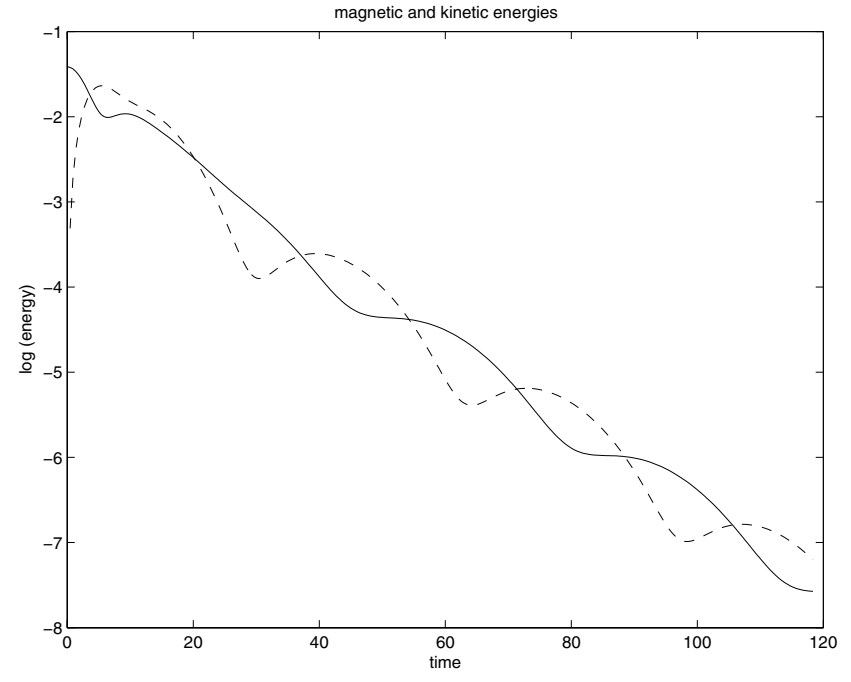

Fig. 3. Energy decay (solid line for the kinetic energy and dashed line for the magnetic energy) with $\eta=10^{-3}, v=10^{-4}$. In contrast to Fig. 2, the decay is dominated by oscillatory exponential damping in which energy equipartition is maintained.

\section{Analytic shear wave description}

\subsection{Introduction}

For a theoretical interpretation of the wave energy losses we need to determine how phase mixing can accelerate the viscous and resistive damping of the axial fields. We must also account for decay profiles which include:

- oscillatory relaxation in which energy equipartition is maintained;

- an asymptotic exponential relaxation in which the kinetic and magnetic energies remain well separated.

We begin by pointing out that a formally exact solution is possible in the special case $\eta=v$, which provides a good approximation to the initial wave evolution even when these coefficients have disparate magnitudes. We go on to explain how, for $v>\eta$, the differing symmetries of the $Z$ and $W$ fields can lead to a separation of the magnetic and kinetic energies during late stages of the decay.

\subsection{Initial decay phase}

The ideal solution derived in Sect. 2.3 shows an oscillatory behaviour along the $y$-direction with the wave number $m t$. If $v$ and $\eta$ are small, we may expect that the form of this ideal solution remains valid at least for short times. This suggests that we seek solutions of the full system by taking

$\left(\begin{array}{c}W(y, t) \\ Z(y, t)\end{array}\right)=\exp (\mathrm{i} k(t) y)\left(\begin{array}{c}W_{0}(t) \\ Z_{0}(t)\end{array}\right)$

where $Z_{0}$ and $W_{0}$ are complex amplitudes.

Although solutions of this form are generally not useful for describing the asymptotic decay, our results show that the initial wave development invariably accounts for the bulk of the energy dissipation due to phase mixing. At longer times the dissipation rate may slow due to the development of either selfsimilar modes or exponentially decaying modes as $t \rightarrow \infty$.

\subsubsection{Exact solution in the case $v=\eta$}

An important special case occurs when the viscous and resistive coefficients are equal. Although this situation is rather academic, it leads to an exact solution which provides a useful approximation even when the damping coefficients are no longer comparible. To derive the solution, we return to system (7)-(8) and note that the function $F^{+}=W+Z$ and $F^{-}=W-Z$ satisfy the relations

$F_{t}^{+}-y F_{x}^{+}=v^{+} \nabla^{2} F^{+}+v^{-} \nabla^{2} F^{-}$

$F_{t}^{-}+y F_{x}^{-}=v^{+} \nabla^{2} F^{-}+v^{-} \nabla^{2} F^{+}$

where

$v^{ \pm}=\frac{v \pm \eta}{2}$

These equations decouple when $\eta=v$ and by assuming a solution of the form

$F^{ \pm}=A^{ \pm}(t) \exp (\mathrm{i} m x+\mathrm{i} k(t) y)$

we find that the wave number $k$ must grow linearly with time according to

$k(t)=k_{0} \pm m t$

where $k_{0}$ is the initial wave number and the plus or minus sign is coherent with the functions $F^{ \pm}$themselves. The amplitudes decay according to

$\dot{A}^{ \pm}(t)=-v^{+} k(t)^{2} A^{ \pm}=-v^{+}\left(k_{0} \pm m t\right)^{2} A^{ \pm}$,

and so we deduce that

$A^{ \pm}(t)=A_{0}^{ \pm} \exp \left[-\frac{v^{+}}{3} \frac{\left( \pm k_{0}+m t\right)^{3}}{m}\right]$.

Whatever values $A_{0}^{ \pm}$and $k_{0}$ may take, the $W$ and $Z$ fields, formed by superposing the $F^{+}$and $F^{-}$functions, possess an exponentially decaying term of the form:

$\exp \left[-\frac{v^{+}}{3} m^{2} t^{3}\right]$

for long enough time $t \gg 1$. Thus the dissipation rate reaches its maximum when $\ddot{A}=0$ which occurs at a time

$\tau_{m}=\left(\frac{2}{v^{+} m^{2}}\right)^{1 / 3}$.

At this time the logarithmic gradient is clearly

$\left|\frac{\dot{A}}{A}\right| \simeq v^{+} m^{2} \tau_{m}^{2}=2^{2 / 3} v^{+1 / 3} \mathrm{~m}^{2 / 3}$.

and the wave length in the $y$-direction has decreased to

$\lambda=\lambda_{m} \simeq m \tau_{m} \simeq v^{+1 / 3} m^{-1 / 3}$.

We conclude that the maximum decay rate of the field is only weakly dependent on the dissipation coefficient $v^{+}$. In particular, the timescale associated with the energy decay scales as $\left(v^{+}\right)^{-1 / 3}$, which translates to around 30 Alfvén times in a typical coronal plasma. 

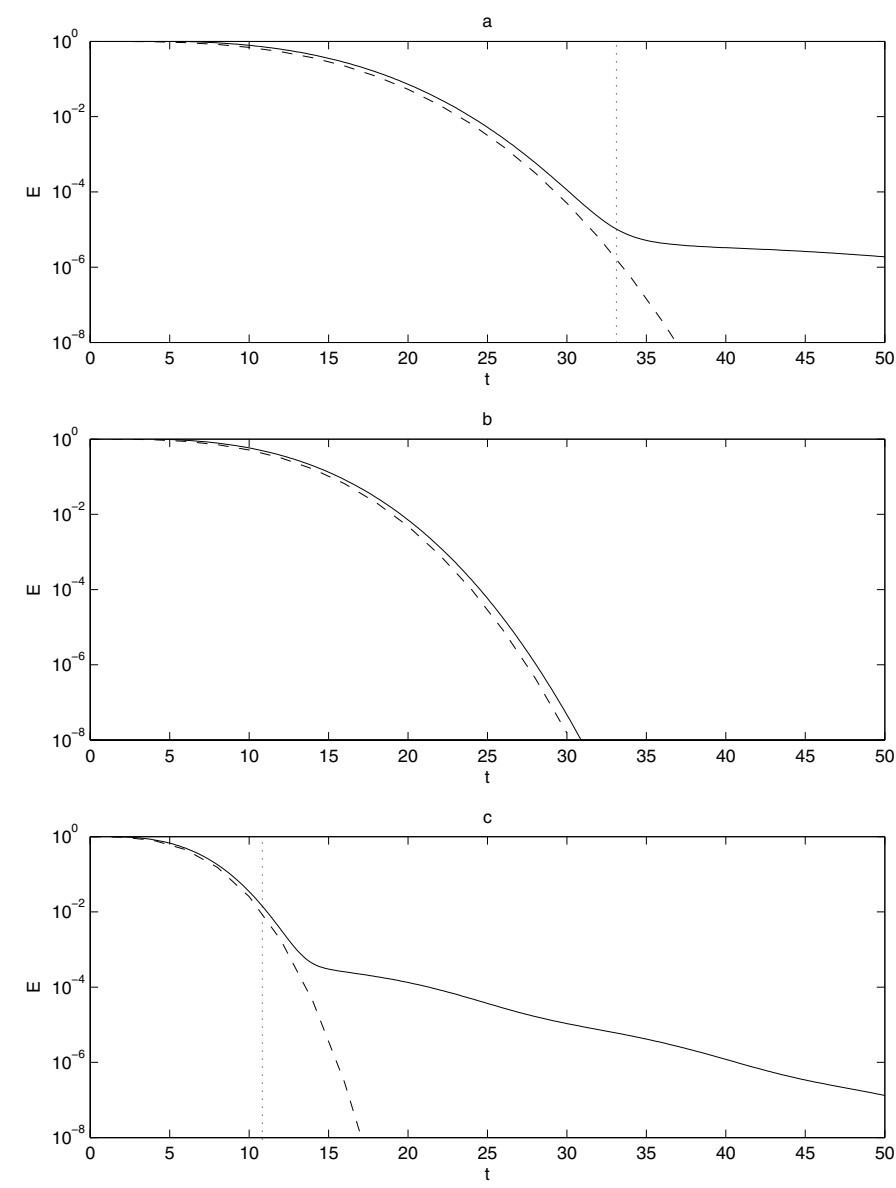

Fig. 4. Initial decay of the total energy for $m=10$ and $v=10^{-5}$ and a) $\eta=10^{-6}$ b) $\eta=10^{-5}$ c) $\eta=10^{-4}$. Comparison between numerical (solid lines) and analytic (dashed lines) expression (23). Both expressions match for times shorter than $\tau_{\mathrm{b}}$ shown as a dotted line. In the case $\eta=v \tau_{\mathrm{b}}$ is infinite.

\subsubsection{Approximate solution for the initial decay}

Although the previous solution is exact only in the special case $\eta=v$, it can be used to describe the initial field decay in more general circumstances. When $v^{-}=(v-\eta) / 2$ is non vanishing, extra terms involving derivatives of the form $v^{-} \nabla^{2}$ are present but these compromise the solution only for sufficiently large times. More precisely, since the second derivative in $y$ is formally equivalent to a multiplication by $k^{2}$, the previous solution (23) should remain good as long as $v^{-} k^{2} \ll 1$, that is for times

$t<\tau_{\mathrm{b}}=\left(\frac{1}{m^{2} v^{-}}\right)^{1 / 2}$.

Since $\tau_{\mathrm{b}}$ always exceeds $\tau_{\mathrm{m}}$ the approximate solution based on Eq. (23) should hold for arbitrary $v$ and $\eta$ beyond the maximum dissipation time.

Figure 4 illustrates the initial decay of the total energy for a fixed viscosity set to $v=10^{-5}$ and various resistivities (from top to bottom $\eta=0.1 v, v, 10 v$ ). The solid lines correspond to the numerical solution of the initial system (11)-(12) whereas the crosses represent the approximate solution (23) with $m=10$. The two curves match perfectly during the initial stages but they remain close together for long times only in the
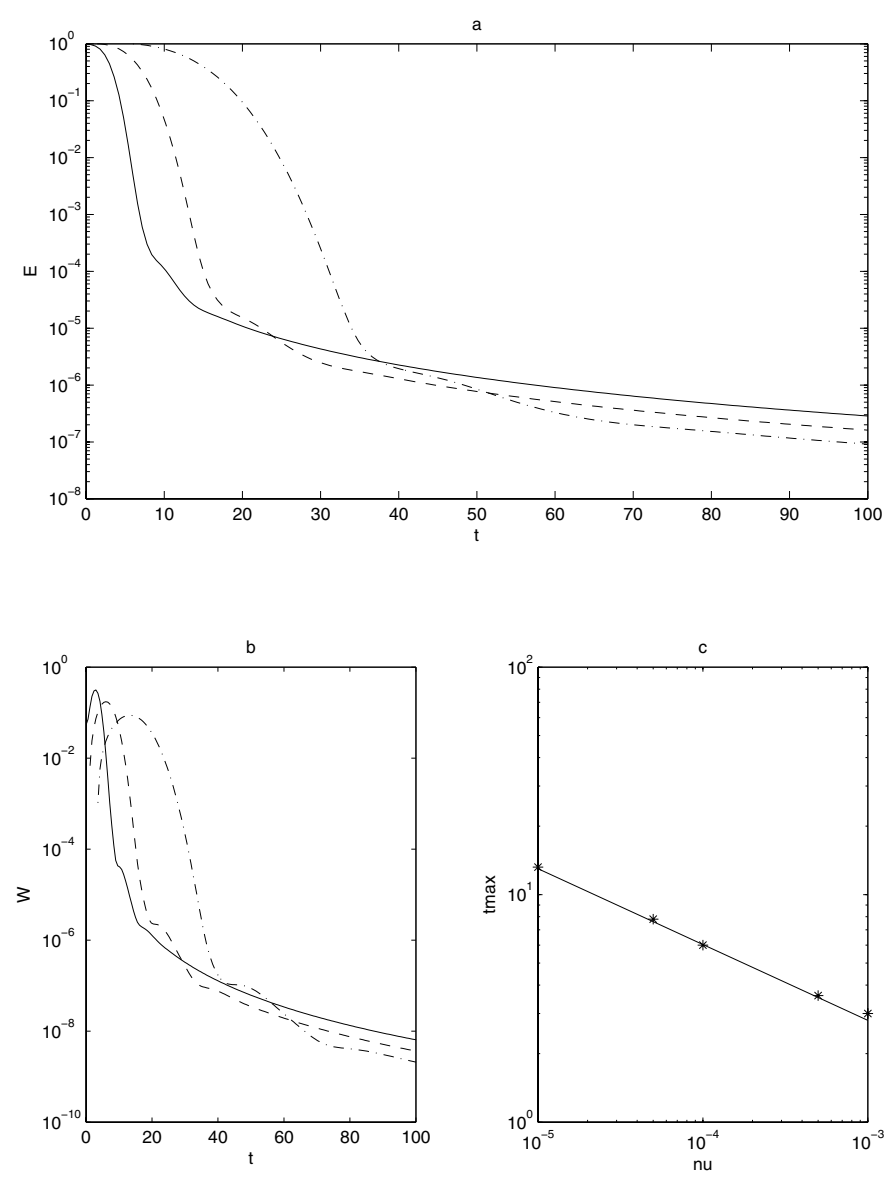

Fig. 5. a) Decay of the total energy for $m=10, \eta=0$ and solid line: $v=10^{-3}$, dashed line: $v=10^{-4}$, dot-dashed line: $v=10^{-5}$. b) Dissipation rate $\mathcal{W}=-\mathcal{E}_{t}$ versus time for the same values of $v$, c) time of the maximum dissipation rate versus the viscosity (logarithmic scales). The straight line of slope $-1 / 3$ fits the data.

case where $\eta=v$. When the diffusion coefficients are well separated, a radical change of behaviour occurs in the decay rate at a time of the order of $\tau_{\mathrm{b}}$ mentioned above. This behaviour will be analyzed in more detail below.

For the moment we emphasize that it is the initial decay $t<\tau_{\mathrm{b}}$ which is effective in removing the bulk of the disturbance field energy within twenty or thirty Alfvén times. Previous phase mixing studies have argued that exponential decay such as Eq. (24) controls the later decay which sets in for myt $\gg 1$ (see e.g. Heyvaerts \& Priest 1983). In fact our results confirm that fast exponential decay can be guaranteed only in the early stages of damping - it breaks down at longer times, at least in physically realistic cases $v>\eta$.

\subsection{The case of purely viscous decay}

\subsubsection{The initial decay phase for $\eta=0$}

Let us analyze more thoroughly the case where the resistivity is turned off. Although the total energy in the system decreases initially according to Eq. (24) with $v^{+}=v / 2$, the analytic description is expected to break down for times $t>\tau_{\mathrm{b}}$. Figure 5 summarizes the different conclusions of Sect. 3.2 in the particular case where $\eta=0$ and $v=10^{-3}, 10^{-4}$ and $10^{-5}$. Panel a 


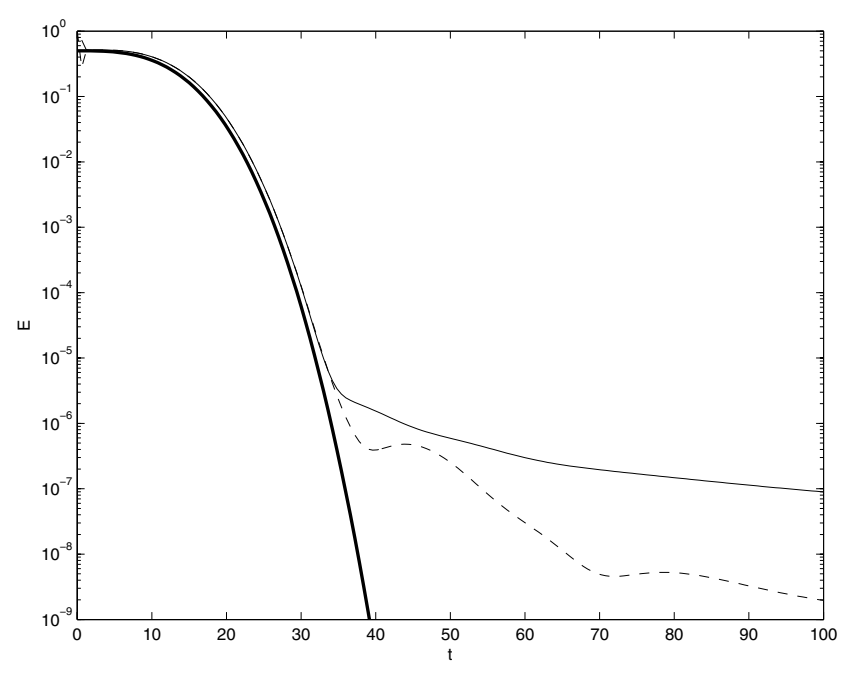

Fig. 6. Separation of the kinetic (dashed line) and magnetic energy (solid line) in the case where $v=10^{-5}$ and $\eta=0$ and $m=10$. The thick line is the analytic expression (23) of the initial stage.

shows the total energy decay and panel $b$ displays the dissipation rate $\mathcal{E}_{t}$ on a $\log$ scale. The latter reaches a maximum for a time $\tau_{\mathrm{m}}$ which scales as $v^{-1 / 3}$ (see panel $\mathrm{c}$ and Eq. (25)). It is also interesting to note that the asymptotic tail in the energy damping does not depend on the viscosity. This point could be important in the case of permanently driven oscillations. Our present purpose, however, is to show that this later phase is associated with the emergence of a self-similar mode whose development promotes a separation between the magnetic and kinetic energies of the plasma observed in Fig. 6 just after the dissipation rate has reached its maximum.

\subsubsection{Breakdown of the wave solution}

When $\eta=0$ system (11)-(12) can be simplified into a second order differential equation for the velocity field

$W_{t t}=-m^{2} y^{2} W+v W_{y y t}$.

Naturally, a similar equation holds for the $Z$-field under the assumption of negligible viscosity (with $\eta$ replacing $v$ and $Z$ replacing $W$ ). These expressions neglect the weak $x$-dependence of $\nabla^{2}$ operator and represent a considerable simplification of the fourth order equation that applies for arbitrary $\eta$ and $v$ (see the appendix). Even so, an exact analytic treatment of (29) is not possible.

By equating successively each pair of terms appearing in this equation, three spatial scales may be identified, namely

$\ell_{\mathrm{i}}=\frac{1}{m t}, \quad \ell_{\mathrm{d}}=(v t)^{1 / 2}, \quad \ell_{\mathrm{s}}=\left(\frac{v}{m^{2} t}\right)^{1 / 4}$.

The first scale reflects ideal wave propagation and arises from the continual build-up of gradients in the $y$-direction in the absence of diffusion. Note that by equating $\ell_{\mathrm{i}}$ with the diffusive scale $\ell_{\mathrm{d}}$, we obtain a time

$\tau \simeq \tau_{\mathrm{m}} \simeq v^{-1 / 3} m^{-2 / 3}$ which scales as the dissipation timescale (25). We conclude that the dissipation of the initial perturbation is maximum when the ideal scale and the diffusion scale are of the same order.

It is interesting to note that Eq. (31) also defines the expected "return-time" for a localized disturbance, launched from the outer boundary. This follows from the observation of Fig. 7 that once strong cross field "corrugations" of wavelength $\lambda_{\mathrm{m}}$ have formed due to phase mixing, diffusion acting on the timescale $\lambda_{\mathrm{m}}^{2} / v \simeq \tau_{\mathrm{m}}$ becomes an effective conduit for propagating information between the outer boundary and the neutral line. This is true no matter whether viscosity or resistivity provides the dominant damping mechanism. It follows that $\tau_{\mathrm{m}}$ should determine the period of oscillatory wave modes set up between the neutral line and the outer boundary $y=1-$ assuming such modes develop. The oscillation periods observed in Fig. 2, do indeed follow the scaling predicted by $\tau_{\mathrm{m}}$ under the replacement $v^{+} \rightarrow \eta$. When viscosity is the primary mechanism however, such modes seem to be suppressed by other viscous phenomena operating in the vicinity of the neutral line. The presence of the scale $\ell_{\mathrm{s}}$ already indicates that a further mechanism may be important close to the neutral sheet.

Recall that, according to the initial symmetries, the $W$-field and its associated time derivatives always become small as $y \rightarrow$ 0 (Fig. 7). The scale $\ell_{\mathrm{s}}$ derives from the assumption that $W_{t t}$ is negligible in Eq. (29). We thus obtain a self-similar mode based on a balance between the diffusive and advective terms. This mode should come into play in the inner field region $y<$ $y_{\mathrm{m}}=\lambda_{\mathrm{m}}$. Since $\ell_{\mathrm{s}}>\ell_{\mathrm{i}}$ for $t>\tau_{\mathrm{m}}$, the self-similar mode can be expected to emerge for sufficiently long times as shown in the last panels of Fig. 7 .

\subsubsection{The self-similar mode}

The previous argument suggests that, if advection and diffusion can be balanced in a localized region surrounding the origin, then self-similar behaviour may be possible based on the variable

$\xi=\frac{m^{2}}{v} y^{4} t$

It follows that, for sufficiently long times, solutions of Eq. (29), i.e. of the original system for $v \gg \eta$, can be constructed of the form

$W=a(t) f(\xi), \quad Z=b(t) g(\xi)$,

where $a(t)$ and $b(t)$ account for very weak variations in the wave amplitudes. We shall find that the self-similar development undoes the equipartition associated with the wave solution and promotes a preferential power-law damping of the $W$-field.

We substitute Eq. (33) in the original system assuming $W_{t}$ to be negligible. The resulting system, namely $m y Z=v W_{y y}$ and $Z_{t}=m y W$, now reduces to four equations: the time dependent terms are given by

$\frac{b}{a}=\alpha m v^{1 / 3} t^{3 / 4}, \quad \frac{\dot{b}}{b}=\frac{\beta}{t}$, 

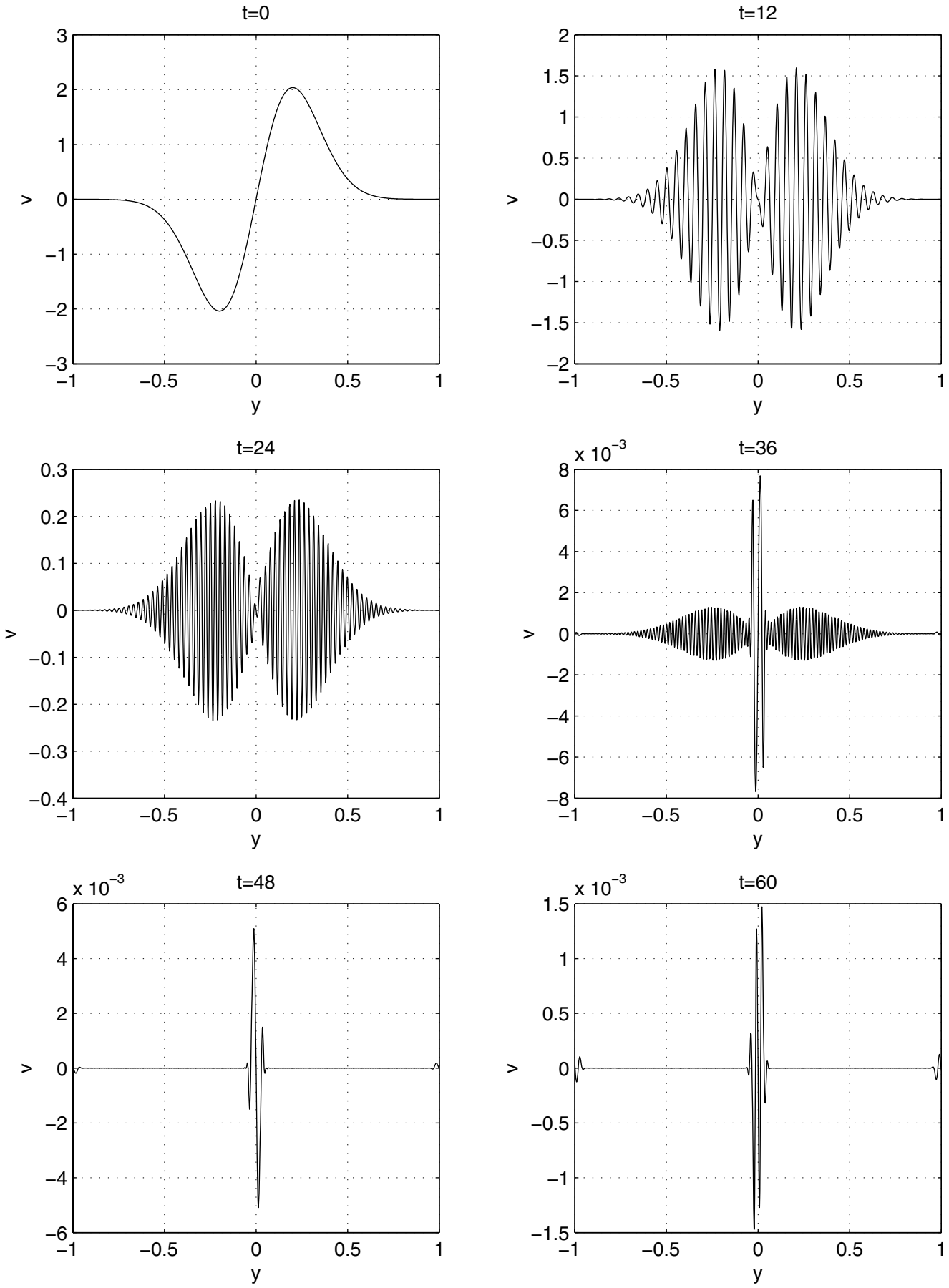

Fig. 7. Temporal evolution of the velocity field as a function of $y$ for $v=10^{-5}$ and $m=10$. As time goes on, more and more corrugations are building up until the wavelength matches the diffusion scale between 30 and 40 Alfvén times when efficient damping occurs. Only a small perturbation concentrated around the null point remains thereafter (self-similar mode).

where $\alpha$ and $\beta$ are separation constants to be determined; also $f(\xi)$ and $g(\xi)$ are related by

$\left(\xi^{\frac{3}{4}} f^{\prime}\right)^{\prime}=\frac{\alpha}{16} \xi^{-1 / 2} g, \quad g^{\prime}+\beta g=\frac{\xi^{\frac{1}{4}}}{\alpha m} g$.

The first of Eq. (34) immediately confirms that the magnetic field decays more slowly than the velocity field by the factor $t^{3 / 4}$.
To obtain more detailed information we exploit the fact that since the field disturbance is an even function of $y$ it has the local expansion

$g(\xi)=g_{0} \xi^{1 / 2}+g_{1} \xi+O\left(\xi^{3 / 2}\right)$

Substituting this expression in the first of Eq. (35) now yields

$f(\xi)=f_{0} \xi^{1 / 4}+\frac{\alpha}{20} g_{0} \xi^{5 / 4}+g_{1} O\left(\xi^{7 / 4}\right)$. 


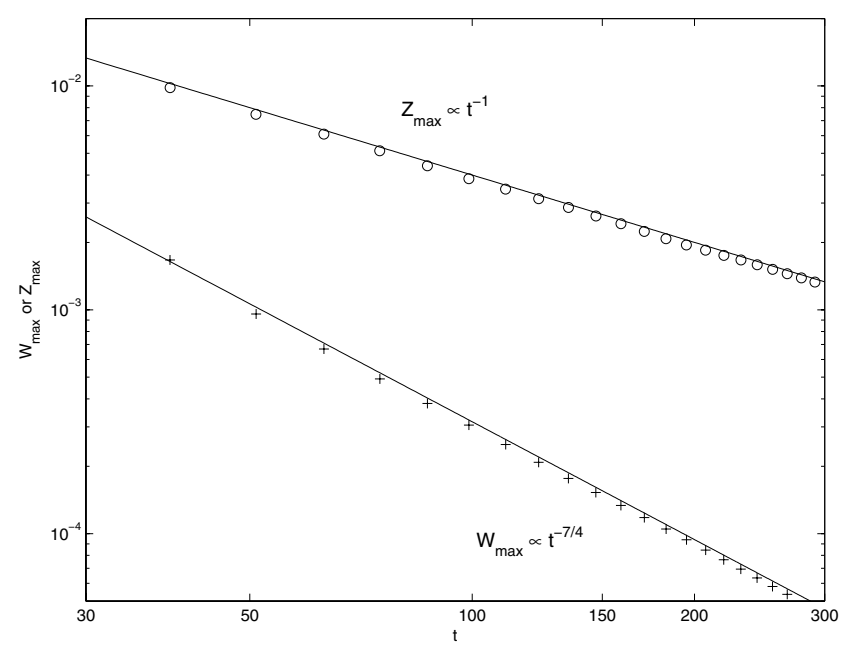

Fig. 8. Linear fit of the maximum velocity (crosses) and maximum magnetic field (circles) against time in a $\log -\log$ plot. The straight lines have slopes $-7 / 4$ for the velocity and -1 for the magnetic field illustrating that these physical quantities vary with time according to the power-laws (39).

This expression has the correct (odd) symmetry but the expected term in $\xi^{3 / 4}$ is clearly missing.

Introducing the expansions for $f$ and $g$ into the second of Eq. (35) gives, on equating similar powers,

$\beta+\frac{1}{2}=\frac{1}{\alpha m} \quad$ and $\quad(1+\beta) g_{1}=0$.

So, provided only that $g_{1}$ is nonvanishing, we must have $\beta=-1$ and $\alpha=-2 / m$ from which it follows that $a$ and $b$ decay with time according to the power-laws

$a(t) \propto t^{-7 / 4} \quad$ and $\quad b(t) \propto t^{-1}$

A key point is that the coefficient $v$ cancels out in the previous analysis and so the evolution of the self-similar mode is independent of the viscosity. These are precisely the properties we observe in the numerical results.

More specifically, the magnetic and kinetic energies should begin to separate very close to the time of maximum dissipation and, from this time onwards, the ratio of the global kinetic to the global magnetic energy should approximate the power law $t^{-3 / 2}$. A further prediction is that fixed points on the wave profile (for example the maximum of $W$ ) should migrate inwards towards the origin according to $y \sim(v / t)^{1 / 4}$. All these characteristics are present on the numerical solution of Fig. 8. This log-log diagram represents the amplitude of the $W$-field (crosses) and $Z$-field (circle) against time. The straight line that fits the data have slopes of $-7 / 4$ and -1 respectively.

Finally, we should also mention that a self-similar variable, namely $\xi=m^{2} \eta /\left(y^{4} t\right)$ can also be associated with the case of finite resistivity but negligible viscosity. In this case however, the influence of the self-similar mode is compromised by the fact that $Z$ and associated time derivatives do not vanish as $y \rightarrow$ 0 . As a result, the oscillatory tendency of the axial field persists for times $t \gg \tau_{\mathrm{m}}$.

\subsection{Monotonic exponentially decaying solutions}

When $v$ and $\eta$ are both finite we recover a final possibility, namely exponentially decaying modes in the limit of large times. To see how this occurs let us assume the ordering $v>$ $\eta>0$ and seek solutions, localized to the origin, which decay as $\exp (-\alpha t)$ where $\alpha$ is a small real number. The system becomes

$-\alpha W+m y Z=v W_{y y}$,

$-\alpha Z-m y W=\eta Z_{y y}$,

on neglecting $v m^{2} W$ from (11) and $\eta m^{2} Z$ from Eq. (12).

The key observation is that for $\alpha$ small enough there is a hybrid length scale given by

$\ell_{\mathrm{h}}=\left(\frac{\eta v}{m^{2}}\right)^{1 / 6}$

which determines a visco-resistive scale. We assume this scale defines a weakly decaying inner solution in the region $y \leq y_{\mathrm{h}}=$ $O\left(\ell_{\mathrm{h}}\right)$.

At the origin we can normalize the fields so that $Z(0)=1$ and $W(0)=0$. Therefore we neglect $W$ in Eq. (41) and solve $-\alpha Z=\eta Z_{y y}$ to obtain an inner approximation for $Z$, namely

$Z(y)=\cos \left(\sqrt{\frac{\alpha}{\eta}} y\right), \quad y \leq y_{\mathrm{h}}$.

If $Z$ is to vanish on the inner scale we require

$\alpha \simeq \frac{\pi^{2}}{4} \eta^{2 / 3} v^{-1 / 3} m^{2 / 3}, \quad(v \gg \eta)$.

An inner solution can now be obtained for $W$ using $v W_{y y} \simeq$ $m y Z$, namely

$W \simeq \frac{m}{v \kappa^{2}}\left(y \cos (\kappa y)-\frac{2}{\kappa} \sin (\kappa y)\right), \quad \kappa=\frac{\pi}{2}(\eta v)^{-1 / 6}$.

This construction yields a monotonic decay rate which coincides with the peak rate (26) when $v$ and $\eta$ are comparable in magnitude. More generally, as reflected by the late decay of Fig. 2, the monotonic decay is considerably slower than the peak rate. A further prediction is that the ratio $|W / Z|$ should scale as $m(\eta / v)^{1 / 2}$.

In general, provided that the ordering $v>\eta>0$ is respected, the exponential mode can be expected to compete for dominance with the self-similar mode during the late decay. But whereas the self-similar analysis remains valid only if the resistive influence is negligible, the emergence of the exponential mode requires that resistive diffusion is sufficiently effective. If we take $\tau_{\mathrm{b}}(28)$ as an upper limit for the resistive time scale, then we obtain $\alpha^{-1} \leq \tau_{\mathrm{b}}$ or $\eta=\eta_{\min } \geq m^{1 / 2} v^{5 / 4}$ as the condition for the exponential mode to be dominant.

This interpretation is borne out by Fig. 9, which shows time plots of kinetic and magnetic energy ratios at various resistivities (with $v>\eta$ fixed at $10^{-4}$ ). We see that when $\eta$ lies outside the narrow window $v>\eta \geq \eta_{\min }$ the ratio is no longer constant in time but follows the $t^{3 / 2}$ divergence predicted by the self-similar mode. 


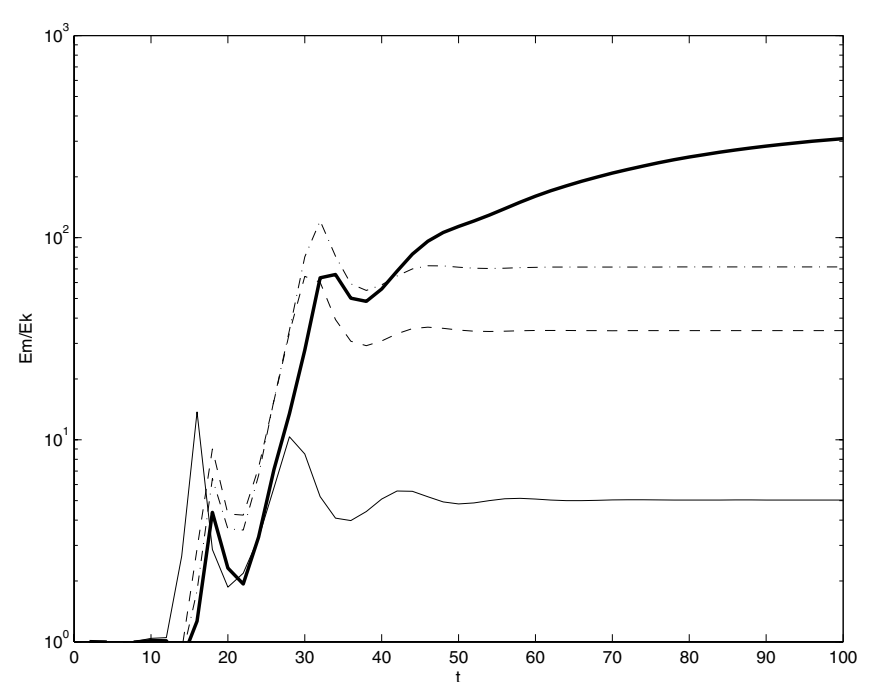

Fig. 9. Plot of the ratio (magnetic energy/kinetic energy) versus time for $v=10^{-4}$ and various resistivities: $\eta=0.5 v$ (thin solid), $\eta=0.1 v$ (dashed), $\eta=0.05 v$ (dot-dashed) and $\eta=0.01 v$ (thick line). This ratio tends to a constant (monotonic decay) scaling as $v / \eta$ for long enough times. But if $\eta$ is much less than $v$, the self-similar mode emerges for $t>\tau_{\mathrm{b}}$ and squeezes out the monotonic decay.

\subsection{Summary}

We have seen that although the bulk of the wave energy in the current sheet channel damps on the timescale $(\eta+v)^{-1 / 3}$, the later decay phases show significant departures from simple exponential damping. In the physically realistic case $v \gg \eta>0$, the wave decay reflects the interplay between several distinct length scales, namely $\ell_{\mathrm{i}}, \ell_{\mathrm{d}}, \ell_{\mathrm{s}}(30)$ and $\ell_{\mathrm{h}}(42)$. The balance $\ell_{\mathrm{i}} \simeq \ell_{\mathrm{d}}$ applies only at the end of the initial stage. In the later stages a self-similar mode develops (on the scale $\ell_{\mathrm{s}} \sim(v / t)^{1 / 4}$ ) which primarily damps the kinetic energy of the plasma, allowing a power law $t^{-3 / 2}$ separation of the magnetic and kinetic energies. The self-similar mode emerges however, only if resistive effects are effectively negligible. If $\eta$ is sufficiently close to $v$, the final stage of the relaxation is dominated by monotonic exponential decay on the visco-resistive scale $\ell_{\mathrm{h}}=\left(\mathrm{v} \eta / \mathrm{m}^{2}\right)^{1 / 6}$. The remnants of the initial wave energy now resides mainly in the magnetic energy of the plasma which dominates the kinetic energy by a factor of approximately $v / \eta$.

\section{Conclusion}

We have considered visco-resistive energy dissipation of shear Alfvén waves within a one-dimensional current sheet. The problem is formulated as an initial value problem in which a finite amount of energy is injected into the current layer. In agreement with previous phase mixing studies (Heyvaerts \& Priest 1983), our analysis implies that the bulk of the wave energy is damped on the timescale $\tau_{\mathrm{m}} \simeq(\eta+v)^{-1 / 3}$. We have stressed however, that the bulk of the damping occurs during the initial stages and that the later decay depends critically on whether viscosity or resistivity provides the dominant damping mechanism. Thus, the detailed evolution requires an understanding of the role played by several disparate length scales in the problem (Sect. 3.3).

An exact solution, derived in Sect. 3.2 and valid for $\eta=v$, provides a useful "transition model" for understanding how the system changes with the relative magnitudes of the dissipation coefficients. This solution makes explicit how the smoothing effects of visco-resistive diffusion balance the tendency for phase mixing to build up sharp cross-field gradients. This balance also controls the initial evolution of the system in the case where $\eta \gg v$. However, oscillations of period $\tau_{\mathrm{m}}$ emerge during the later phases $\left(t>\tau_{\mathrm{m}}\right)$ and the decay is characterized by exponentially damped, oscillatory modes which maintain equipartition in the global kinetic and magnetic energies.

The decay profile is considerably richer in the physically realistic case where $v \gg \eta$. The initial evolution again mimics the exact solution, but a self-similar mode develops for $t>\tau_{\mathrm{m}}$ which damps mainly the kinetic energy of the plasma and provides a power law separation $\left(t^{-3 / 2}\right)$ in the magnetic and kinetic components. When $\eta$ and $v$ are sufficiently close however, self-similar behaviour is superceded by monotonic exponential damping on the hybrid length scale $\ell_{\mathrm{h}}=(\eta v)^{1 / 6}-$ the viscoresistive scale of Sect. 3.4. Once again a dominant fraction of the residual wave energy $(v / \eta)$ remains in the magnetic field. Although the asymptotic tail plays a minor role in the energetics of this initial value problem, it could become more important in a driven problem (Ruderman et al. 1997). In this case, the asymptotic behaviour may give rise to a permanent regime where visco-resistive dissipation is balanced by a constant supply of energy from the boundaries. This question is currently being investigated.

What implications have these present results for a typical coronal plasma in which $v \gg \eta$ ? As far as energy release is concerned, we note that viscous damping can occur over roughly 40 Alfvén times, which translate to around $100 \mathrm{~s}$ in a typical coronal plasma where $v \simeq 10^{-5}$. The fact that the viscous damping time is comparable to the release time for a solar flare suggests that viscous effects could be important in both active region and solar flare plasmas. By contrast purely resistive phase mixing (as studied by Hood et al. 1997a,b) is considerably less effective: it requires $10^{5}$ s to achieve significant damping for a resistivity of the order of $10^{-14}$, which lies well outside the range for flare energy release.

Despite the very simple field geometry considered in the present work, we have seen that visco-resistive coupling can give rise to quite complex dissipation modes. Preliminary results suggest that similar behaviour - and corresponding global energy loss rates - can be expected for wave dissipation in more general $X$-point geometries. One slight disclaimer is that the Braginskii viscous stress tensor (Braginskii 1965) becomes highly non-isotropic in regions of sufficiently strong magnetic field, an effect not considered in the present analysis, nor in previous models of phase-mixing. However, our central results should remain valid since the inequality $v \gg \eta$ still holds even when the reduced transverse viscosity is taken into account. To what extent the efficiency of viscous dissipation depends on the details of the magnetic field geometry is currently being studied. 
Acknowledgements. This work was supported by the Marsden Fund (02-UOW-050 MIS).

\section{Appendix}

For completeness, we derive here the fourth order differential equation that must be satisfied by the $W$-field or the $Z$-field. We start from the system (11)-(12) and neglect the $\mathrm{vm}^{2}, \mathrm{\eta m}^{2}$ terms:

$W_{t}+m y Z=v W_{y y}$,

$Z_{t}-m y W=\eta Z_{y y}$.

By eliminating $Z$ in these equations it is straightforward to show that $W$ satisfy the equation

$$
\begin{array}{r}
v \eta y^{2} W_{y y y y}-2 v \eta y W_{y y y}+\left(2 \eta v W-(\eta+v) y^{2} W_{t}\right)_{y y} \\
+2 \eta y W_{y t}+y^{2} W_{t t}+m^{2} y^{4} W=0 .
\end{array}
$$

Since the smallest coefficients multiply the highest derivatives the temptation to neglect terms involving $\eta v$ should be resisted. In fact by introducing the ideal solution (13) in (48), we see that the first term is proportional to $v \eta y^{2} t^{4}$. Although $v$ and $\eta$ are small, this term increases very rapidly with time and we are led to a situation where all the terms in (48) become of the same order, so that no further assumptions may simplify the analysis. Also the null point $y=0$ is a singular point for (48). For these reasons, the differential equation succumbs to a global analytic treatment only in special cases ( $\operatorname{such}$ as $\eta=v$ ). More generally, a numerical treatment is required.

\section{References}

Braginskii, S. I. 1965, Rev. Plasma Phys., 1, 205

Craig, I. J. D., \& McClymont, A. N. 1991, ApJ, 371, L41

Craig, I. J. D., \& McClymont, A. N. 1993, ApJ, 405, 207

Hassam, A. B. 1992, ApJ, 399, 159

Heyvaerts, J., \& Priest, E. R. 1983, A\&A, 117, 220

Hollweg, J. V. 1986, ApJ, 306, 730

Hood, A. W., Gonzalez-Delgado, D., \& Ireland, J. 1997a, A\&A, 324, 11

Hood, A. W., Ireland, J., \& Priest, E. R. 1997b, A\&A, 318, 957

Priest, E. R., \& Forbes, T. 2000, Magnetic Reconnection (Cambridge U Press)

Ruderman, M. S., Goossens, M., Ballester, J. L., \& Oliver, R. 1997, A\&A, 328, 361

Ruderman, M. S., Nakariakov, V. M., \& Roberts, B. 1998, A\&A, 338, 1118

Spitzer, L. 1962, Physics of Fully Ionized Gases (John Wiley \& Sons) 